\title{
THE SCIENCE PROGRAM OF THE HUBBLE SPACE TELESCOPE
}

\author{
Nolan R. Walborn \\ Science Program Selection Office \\ Space Telescope Science Institute \\ 3700 San Martin Drive \\ Baltimore, MD 21218, USA
}

\section{Introduction}

The HST science program is comprised of two principal components: the General Observer (GO) and the Guaranteed Time Observer (GTO) programs. A third component, the Archival Research (AR) program, will become increasingly important as time goes by. The GOs are selected by peer review of competitive proposals from the worldwide astronomical community, in a process managed by the STScI. The initial GO proposal deadline was October 1, 1988 and the corresponding review meetings took place during April 1989. The GTOs are members of the Investigation Definition and Observatory Scientist Teams who were competitively selected by NASA following an Announcement of Opportunity in 1977, to design and build the HST Science Instruments and participate in the project development, and who were awarded a decreasing fraction of the observing time during the initial two to three years of operations to conduct scientific observations with their instruments. The detailed GTO observing programs were received by the STScI on April 1, 1985, and had been revised a number of times since then.

The detection of the unfortunate spherical aberration in the HST primary mirror shortly after its launch on April 24, 1990 had detrimental implications for all of the preexisting science program. The procedures adopted for revising both the GO and GTO programs in response to the optical problem will be briefly described, together with the status of their Cycle 1 implementation and of the subsequent Cycle 2 selection process. Examples of the unique scientific data obtainable with HST even in its current state and the plans for recovering its full originally intended capabilities are discussed elsewhere throughout this session.

\section{Cycle 1}

Following extensive consultations with the various STScI advisory and oversight committees, it was determined that the previously approved GO programs only should be reassessed for the Cycle 1 calendar year. The GOs were given an opportunity to submit proposed readjustments of their programs in view of the optical problem, which were then considered along with STScI technical comments by a subset of the original Telescope Allocation Committee and Subdiscipline Panels, meeting during February 1991. The principal guideline to both the GOs and the TAC was to favor programs which promised greater scientific returns from the current instrument, rather than heroic efforts for marginal results from programs designed for the original specifications. However, qualitative revisions of the original scientific objectives were not permitted. Only a small number of programs were 
withdrawn entirely by their authors, but the time increases requested by the remainder were relatively modest, while the feasibility and scientific viability issues were generally well addressed, so that a substantial majority of the original programs could finally be retained. Of an original total of 165 approved Cycle 1 proposals (including those with supplemental scheduling priority), 112 remain with high priority for a total of 1283 hours of spacecraft time and 262 hours of parallel time, while 25 proposals were placed in an inactive supplemental reserve pool, and 28 were unapproved. There is no guaranteed deferment of previously approved GO programs that were not retained, but they will receive precedence over any similar new proposals, if resubmitted in any subsequent cycle through the first with refurbished instruments.

The readjustment of the GTO program was also extensively discussed among the STScI, NASA, and the appropriate external committees, and the final recommendations have been formulated as NASA Policy 14. It provides for two enhancements of the GTO program: first, an extension to a total of five years or until two years following the first refurbishment mission, whichever occurs later; and second, a time augmentation equivalent to three months of dedicated calendar time distributed through Cycles 2-4, for which the GTOs will compete among themselves, and the proposals for which will be evaluated by the Cycle 2 TAC comparatively with those of the GOs. The total GTO time will not exceed $30 \%$ of the available science time on the average, or $40 \%$ in any given year. GTO programs rendered infeasible by the spherical aberration can be deferred to the initial refurbished instruments, but they do not prevent the TAC from recommending approval of related GO programs in the interim if scientifically and technically justified.

The implementation of the Cycle 1 science program began in earnest during July 1991. A list of the program titles actually executed during the two weeks containing the General Assembly is given in Table 1. It demonstrates the diversity and extent of the HST program, and provides an indication of the substantial results to be expected from the complete Cycle 1 , in comparison with the qualitatively impressive but relatively few earlier scientific data available for presentation here.

\section{Cycle 2}

The proposal deadline for Cycle 2 was August 16, 1991. A total of 483 proposals was received, including 38 GTO/Augmentation and 9 AR. The subdiscipline breakdown is as follows: Galaxies \& Clusters, 74; Quasars \& AGN, 92; Interstellar Medium, 92; Stellar Astrophysics, 135; Stellar Populations, 44; and Solar System, 46. The Principal Investigators are from 19 different countries. The Subdiscipline Panel and final TAC meetings to review them will be held during December 4 through 13, 1991, and it is anticipated that the selected Cycle 2 observations will begin in July 1992. 
TABLE 1-HST SCIENCE PROGRAMS EXECUTED DURING THE GENERAL ASSEMBLY

Program Titles for July 22-A ugust 4, 1991

GTO/IMAGING AND UV SPECTROPHOTOMETRY OF LOCAL-GROUP

PLANETARY NEBULAE

GTO/SPECTROPOLARIMETRY OF MAGNETIC WHITE DWARFS

GTO/PECULIAR AND INTERACTING GALAXIES

GTO/STELLAR-WIND VARIATIONS

GTO/DUST LANES AND FILAMENTARY STRUCTURES IN DOMINANT ELLIPTICAL GALAXIES

GTO/A SEARCH FOR PLANETS AROUND NEARBY STARS

GO/SEARCH FOR WOLF-RAYET STARS IN LOCAL-GROUP GIANT H II REGIONS

GO/CATACLYSMIC VARIABLES AND MILLISECOND PULSARS IN GLOBULARCLUSTER CUSPS

GO/AEROSOLS IN PLANETARY ATMOSPHERES

GTO/SUPERNOVA REMNANTS AND NUCLEOSYNTHESIS

GTO/EVOLUTION OF LYMAN-ALPHA AND C IV ABSORPTION SYSTEMS

GTO/STAR FORMATION REGIONS: HH AND T TAU OBJECTS

GO/DETERMINATION OF THE MASS DENSITIES OF PLUTO AND CHARON

GO/HIGH-RESOLUTION IMAGING OF THE GALACTIC CENTER AT 1 MICRON

GO/THE LUMINOSITY FUNCTION OF THE TRAPEZIUM CLUSTER

GO/LYMAN-ALPHA IMAGING OF YOUNG AND FORMING GALAXIES AT LARGE REDSHIFTS

GTO/IMAGING AND SPECTROSCOPY OF ELLIPTICAL GALAXIES 